\title{
Effect of mesoporous bioactive glass on odontogenic differentiation of human dental pulp stem cells
}

\author{
Lin Zhu ${ }^{1}$, Jingyi Li ${ }^{1}$, Yanmei Dong ${ }^{\text {Corresp. } 1}$ \\ 1 Department of Cariology and Endodontology, Peking University School and Hospital of Stomatology \& National Clinical Research Center for Oral Diseases \\ \& National Engineering Laboratory for Digital and Material Technology of Stomatology \& Beijing Key Laboratory of Digital Stomatology, Beijing, China \\ Corresponding Author: Yanmei Dong \\ Email address: kqdongyanmei@bjmu.edu.cn
}

Healthy pulp tissue plays an important role in normal function and long-term retention of teeth. Mesoporous bioactive glass (MBG) as a kind of regenerative biomaterials shows the potential in preserving the vital pulp. In this study, MBG prepared by organic template method combined with sol-gel method were used in human dental pulp cell culture and ectopic mineralization experiment. Real-Time PCR was used to explore its ability to induce odontogenic differentiation of dental pulp cells. MBG and rat crowns were implanted under the skin of nude mice for 4 weeks to observe the formation of pulp dentin complex. We found that MBG can release Si and $\mathrm{Ca}$ ions and has a strong mineralization activity in vitro. The co-culture of MBG with human dental pulp cells promoted the expression of DMP-1(dentin matrix protein-1) and ALP(alkalinephosphatase) without affecting cell proliferation. After 4 weeks of subcutaneous implantation in nude mice, the formation of hard tissue with regular structure under the material could be seen, and the structure was similar to dentin tubules. These results indicate that MBG can induce the differentiation of dental pulp cells and the formation of dental pulp-dentin complex and has the potential to promote the repair and regeneration of dental pulp injuries. 
1 Effect of mesoporous bioactive glass on odontogenic differentiation of 2 human dental pulp stem cells

3

4 15

Lin Zhu, ${ }^{1 \dagger}$ Jingyi Li, ${ }^{1 \dagger}$ Yanmei Dong ${ }^{*}$.

${ }^{1}$ Department of Cariology and Endodontology, Peking University School and Hospital of Stomatology \& National Clinical Research Center for Oral Diseases \& National Engineering Laboratory for Digital and Material Technology of Stomatology \& Beijing Key Laboratory of Digital Stomatology, Beijing, 100081, PR China $\$$ The first two authors should be regarded as joint First Authors' is permitted if requested. * Corresponding author: Dr. Yanmei Dong, Department of Cariology and Endodontology, Peking University School and Hospital of Stomatology, 22 Zhong-guancun South Avenue, Beijing, 100081, China. E-mail addresses: kqdongyanmei@bjmu.edu.cn 
16

17

\section{Abstract}

Healthy pulp tissue plays an important role in normal function and long-term retention of teeth. Mesoporous bioactive glass $(\mathrm{MBG})$ as a kind of regenerative biomaterials shows the potential in preserving the vital pulp. In this study, MBG prepared by organic template method combined with sol-gel method were used in human dental pulp cell culture and ectopic mineralization experiment. Real-Time PCR was used to explore its ability to induce odontogenic differentiation of dental pulp cells. MBG and rat crowns were implanted under the skin of nude mice for 4 weeks to observe the formation of pulp dentin complex. We found that MBG can release $\mathrm{Si}$ and $\mathrm{Ca}$ ions and has a strong mineralization activity in vitro. The co-culture of MBG with human dental pulp cells promoted the expression of DMP-1(dentin matrix protein-1) and ALP(alkaline phosphatase) without affecting cell proliferation. After 4 weeks of subcutaneous implantation in nude mice, the formation of hard tissue with regular structure under the material could be seen, and the structure was similar to dentin tubules. These results indicate that MBG can induce the differentiation of dental pulp cells and the formation of dental pulp-dentin complex and has the potential to promote the repair and regeneration of dental pulp injuries.

Keywords: Mesoporous bioactive glass; Pulp cells; Dental pulp dentin complex 


\section{Introduction}

Healthy pulp tissue plays an important role in normal function and long-term retention of teeth. The principle of treatment of periapical diseases of pulp should preserve the vital pulp as much as possible and restore the structure and function of the dental pulp-dentin complex. Bioactive glass (BG) is a kind of inorganic material with silicon, calcium and phosphorus as the basic system. Previous studies have confirmed that BG has a good application prospect in the repair and regeneration of skin and bone tissue injuries[1, 2].

Our previous studies have shown that a serials of micro-nano sized BG prepared by sol-gel method can promote migration, proliferation, odontogenic differentiation and mineralization of human dental pulp cells[3]. The rat molars were directly covered with the pulp capping agent containing micro-nano $\mathrm{BG}$, and the formation of a restorative dentine bridge with the dentin tubulo-like structure at the exposed pulp hole was observed at 4 weeks[4, 5]. These BG have uneven particle size distribution, poor dispersion and agglomeration, which to a certain extent limits the performance and clinical application effect of BG. In order to improve the morphology of micro-nano BG and increase its biological activity, the particles can be prepared into porous materials by using sol-gel method combined with specific organic template technology[6]. These porous BG particles with more uniform particle size, smaller pore size and better dispersion can be divided into mesoporous materials. The mesoporous structure in mesoporous bioactive glass (MBG) increases the specific surface area of the material so much that the ions exchange rapidly after contact with the liquid, which is conducive to the release of the bioactive ions and can be decomposed more easily[7]. Yan et al. found that hydroxyapatite could be formed after MBG particles were soaked in SBF solution for 4 hours, which has a stronger ability to promote mineralization in vitro[8]. Meanwhile, MBG can also be used as a carrier to load drugs or growth 
factors to form a local microenvironment that is conducive to the proliferation and differentiation of dental pulp cells. In addition, MBG can also promote the proliferation of osteoblasts/fibroblasts, promote the osteogenic differentiation of bone marrow mesenchymal stem cells, and enhance the activity of alkaline phosphatase[1,9]. However, the effect of MBG on the regeneration of dental pulp dentine complex is still rarely confirmed by relevant studies.

In this study, MBG prepared by organic template method combined with sol-gel method were used in human dental pulp cell culture and ectopic mineralization experiment, to explore its ability to induce odontogenic differentiation of dental pulp cells, and to explore its application prospect in the field of dental pulp injury repair and regeneration.

\section{Methods}

\subsection{Preparation and characterization of MBG}

MBG (molar percentage of chemical composition is $60 \% \mathrm{SiO}_{2}, 36 \% \mathrm{CaO}$ and $4 \% \mathrm{P}_{2} \mathrm{O}_{5}$ ) was prepared by sol-gel method combined with organic template technology. The preparation method was briefly described as follows. Hexadecyl trimethyl ammonium bromide (CTAB; Guangzhou Chemical Reagent Factory; Guangdong; China) was added to a mixture of deionized water and ethanol to form CTAB micelles, and then tetraethyl silicate (TEOS; Guangzhou Chemical Reagent Factory; Guangdong; China) dissolved in cyclohexane was added. Adding ammonia water initiates the reaction to promote TEOS hydrolysis. The mixture was stirred, and triethyl phosphate (TEP; Guangzhou Guanghua Chemical Reagent Co. LTD; Guangdong; China) and calcium nitrate tetrahydrate (CN; Guangzhou Chemical Reagent Factory; Guangdong; China) were added sequentially to form a white precipitate. The precipitate collected by filtration was washed with ethanol and deionized water, and dried at room temperature. Finally, MBG is obtained after 
81

removal of organic matter and nitrate by calcination at $650^{\circ} \mathrm{C}$. The material was prepared by National Engineering Research Center for Tissue Restoration and Reconstruction, South China University of Technology.

Field emission scanning electron microscopy (FE-SEM, S4800, JEOL, Japan) was used to observe the surface structure of MBG. The samples were sprayed with platinum at 50 mTorr for 5 min before being observed by FE-SEM. Transmission Electron Microscope (TEM; JEM-2100HR, JEOL, Japan) was used to observe the pore morphology and internal structure of MBG. In the previous study (nitrogen desorption experiment), the specific surface area of MBG particles was $540.400 \mathrm{~m}^{2} / \mathrm{g}$, and the average pore diameter was about $7.325 \mathrm{~nm}[10]$.

\subsection{Physicochemical properties of $\mathrm{MBG}$}

MBG and 45S5 bioglass were used as the experimental group and the control group for the study of physicochemical properties. 45S5 is a traditional bioactive glass and has been widely used in clinical practice, but it still has some limitations such as strong alkalinity that may cause tissue necrosis, so it is used as a control group to evaluate whether the newly prepared MBG has certain improve.

MBG particles and $45 \mathrm{~S} 5$ bioglass (molar percentage of chemical composition is $45 \% \mathrm{SiO}_{2}$, $24.5 \% \mathrm{CaO}, 6 \% \mathrm{P}_{2} \mathrm{O}_{5}$ and $24.5 \% \mathrm{NaO}$ ) particles were placed in a $180^{\circ} \mathrm{C}$ constant temperature drying oven for sterilization at high temperature for 4 hours. They were added to dulbecco's modified eagle medium (DMEM; Gibco, USA; $\mathrm{pH}=7.40$ ) at concentrations of 0.1 and $1 \mathrm{mg} / \mathrm{mL}$ respectively and shaken at $37^{\circ} \mathrm{C}$ and $120 \mathrm{rpm}$ to obtain homogeneous suspension. At $1,3,6,12$ and 24 hours, $\mathrm{pH}$ values of the suspension in each group were detected with a $\mathrm{pH}$ meter (PH3-3C, yueping Inc, shanghai, China), and $\mathrm{pH}$ curves of each group were drawn. The experiment was 
104

105

106

107

108

109

110

111

112

113

114

115

116

117

118

119

120

121

122

123

124

125

126 3

repeated for 3 times independently.

The sterilized MBG particles and 45S5 BG particles were added into the DMEM at a concentration of $0.1 \mathrm{mg} / \mathrm{mL}$. After being swished at $37^{\circ} \mathrm{C}$ at $120 \mathrm{rpm}$ for 24 hours, the supernatant was centrifuged at $14,000 \mathrm{~g} / \mathrm{min}$ for 5 minutes. After the supernatant was filtered with a $0.22 \mathrm{~m}$ filter, $5 \mathrm{~mL}$ was taken and the concentration of $\mathrm{Si}, \mathrm{Ca}$ and $\mathrm{P}$ ions in each group was tested by inductively coupled plasma analysis (ICP, ICAP 6300, Thermo, German).

The ability of MBG to form hydroxyapatite in vitro was determined by FE-SEM, Fourier Transform Infrared Spectroscopy (FTIR, Nexus, America) and X-Ray Diffraction (XRD, XPert Pro MPD, Panaco, Netherlands). MBG particles were added into the solution of simulated body fluid $\left(\mathrm{SBF}\right.$ ) at a concentration of $1 \mathrm{mg} / \mathrm{mL}$ (the ion concentrations were $\mathrm{Na}^{+} 142 \mathrm{mmol} / \mathrm{L}, \mathrm{K}^{+} 5.0$ $\mathrm{mmol} / \mathrm{L}, \mathrm{Ca}^{2+} 2.5 \mathrm{mmol} / \mathrm{L}, \mathrm{Mg}^{2+} 1.5 \mathrm{mmol} / \mathrm{L}, \mathrm{Cl}^{-} 147.8 \mathrm{mmol} / \mathrm{L}, \mathrm{HCO}_{3}^{-} 1.0 \mathrm{mmol} / \mathrm{L}, \mathrm{SO}_{4}{ }^{2-} 0.5$ $\mathrm{mmol} / \mathrm{L}, \mathrm{pH}=7.25-7.45$ ). After shaking at $37^{\circ} \mathrm{C}$ and $120 \mathrm{rpm}$ for 24 hours, the supernatant was discarded by centrifugation at $3500 \mathrm{~g} / \mathrm{min}$ for 5 minutes. The remaining solid MBG powder was removed. After washing and precipitation with acetone and deionized water for 3 times, the powder was dried at $37^{\circ} \mathrm{C}$ in a vacuum drying oven. The mineral deposition on the surface was observed by FE-SEM. Infrared absorption spectra were measured by FTIR. The XRD pattern was obtained by Xpertpro X-ray diffraction analyzer. $\mathrm{Cu}$ target $\mathrm{K} \alpha$ ray was used to scan the sample from $10^{\circ}$ to $80^{\circ}$, tube voltage was $40 \mathrm{kV}$, tube current was $100 \mathrm{~mA}$. Hydroxyapatite (HA) standard card was used as control to analyze the mineral composition.

2.3 Effects of MBG on proliferation, differentiation of dental pulp cells

MBG particles were sterilized by dry heat in $180^{\circ} \mathrm{C}$ incubator for 4 hours, then cooled to room temperature for later use. The homogeneous suspension was prepared by adding MBG into 
127 anhydrous ethanol at $0.1 \mathrm{mg} / \mathrm{mL}$ and shaken at $37^{\circ} \mathrm{C}$ and $120 \mathrm{rpm}$. The $\mathrm{MBG}$ suspension was

128 added into 96 -well plate and 12 -well plate at the volume of $100 \mu \mathrm{L} /$ well and $1400 \mu \mathrm{L} /$ well,

129 respectively. In the control group, equal volume of anhydrous ethanol was added to the culture

130 plates, and the plates were placed in a UV ultra clean table to be air-dried overnight to ensure that

131 the BG particles was firmly attached to the bottom of the culture plates. The plates were sterilized

132 by ultraviolet radiation for 30 minutes for later use.

133 Human dental pulp cells (hDPCs) were isolated from freshly extracted impacted wisdom teeth

134 of patients with informed consent from the Department of Oral and Maxillofacial Surgery as

135 previously described (PKUSSRB-202053006)[11]. Cells were cultured in DMEM containing 10\%

136 fetal calf serum (FBS; Kangyuan, Tianjin, China), 1\% penicillin and streptomycin (Gibco) in the

137 incubator at $37^{\circ} \mathrm{C}$ and $5 \% \mathrm{CO}_{2} .0 .25 \%$ trypsine-EDTA solution was used for digestion when the

138 cell reached $80 \%$. The cell culture medium was changed every 2 days. Cells in passages $4-6$ were

139

140

141

142

143

144

145

146

147

148

149 used for all the experiments.

The proliferation of hDPCS was detected by MTT assay. The cells were seeded at the density of $3 \times 10^{3}$ cells/well in 96-well plates (Corning, America) pretreated with MBG suspension or anhydrous ethanol, with 5 wells per group. The cells were incubated at $37^{\circ} \mathrm{C}$ and $5 \% \mathrm{CO}_{2}$, and the liquid was changed every other day. On days $1,3,5$, and 7, $180 \mu \mathrm{L}$ fresh DMEM and $20 \mu \mathrm{L}$ MTT (Sigma, America) solution at a concentration of $5 \mathrm{mg} / \mathrm{mL}$ were added to each well for $4 \mathrm{~h}$ at $37^{\circ} \mathrm{C}$ and 5\% $\mathrm{CO}_{2}$. After that, $150 \mu \mathrm{L}$ DMSO (Sigma) was added to each well and the plates were shaken at $37^{\circ} \mathrm{C}$ for at least 10 min until the crystals were dissolved. The optical density (OD) was measured at $490 \mathrm{~nm}$ by a microplate analyzer.

HDPCs were seeded in 12-well plates pretreated with anhydrous ethanol or MBG suspension at a density of $1 \times 10^{5}$ per well and cultured at $37^{\circ} \mathrm{C}$ and $5 \% \mathrm{CO}_{2}$. After 7 days, Total RNA was

Peer) reviewing PDF | (2021:06:63004:1:0:NEW 25 Sep 2021) 
150 extracted using Trizol (TRIzol, Invitrogen) following manufacturer's instructions. Using reverse 151 transcription kit, cDNA was synthesized by the Prime Script RT Master Mix (Takara, Tokyo, 152 Japan). Target genes were dentin matrix protein (DMP-1) and alkaline phosphatase (ALP), 153 glyceraldehyde-phosphate dehydrogenase (GAPDH) was used as internal reference, and primer sequences were shown in Table $1.20 \mu \mathrm{L}$ of amplification reaction system was pre-denaturated and 155 activated at $95^{\circ} \mathrm{C}$ for $3 \mathrm{~min}$, denaturated at $95^{\circ} \mathrm{C}$ for $3 \mathrm{~s}$, and annealed and extended at $60^{\circ} \mathrm{C}$ for 20 $156 \mathrm{~s}$ for 40 cycles in total in the ABI QuantStudio 3 Real-Time PCR.

Table 1. Primer sequences

\begin{tabular}{ll} 
Gene & Sequence $\left(\mathbf{5}^{\prime} \mathbf{- 3} \boldsymbol{\prime}\right)$ \\
\hline ALP & Forward: AGCACTCCCACTTCATCTGGAA \\
& Reverse: GAGACCCAATAGGTAGTCCACATTG \\
& Forward: AGGAAGTCTCGCATCTCAGAG \\
DMP-1 & Reverse: TGGAGTTGCTGTTTTCTGTAGAG \\
& Forward: GAAGGTGAAGGTCGGAGTC \\
GAPDH & Reverse: GAGATGGTGATGGGATTTC
\end{tabular}

2.4 MBG induced the formation of dental pulp dentine complex in rats University (LA2011-057). All the experimental animals were obtained from the Beijing Vital River Laboratory Animal Technology Co., Ltd. The experimental process and the animal's feeding, nursing and living conditions are all in the SBF environment. Four male nude mice in one cage were included in this observational experiment. 
167 the rats was soaked in $75 \%$ alcohol for 10 seconds, the upper and lower jaws were surgically

168

169

170

171

172

173

separated, and sterile PBS was washed for 3 times. The upper and lower first molars were separated from the jaws and rinsed with sterile PBS for 3 times. The root of the molar was removed from the neck of the tooth with a surgical blade, and the crown and pulp tissue were preserved. The molar was placed in fresh DMEM medium for use. The sterilized MBG particles (2mg) were covered in the crown pulp section as the experimental group, and the crown implant alone as the control group. Four samples were set in each group.

Male BALB/c nude mice aged 6 weeks were anesthetized by intraperitoneal injection of 0.5 $\%$ pentobarbital sodium $30 \mathrm{mg} / \mathrm{kg}$. The surgical area was disinfected with $75 \%$ alcohol, and $3 \mathrm{~mm}$ transverse incisions were made on both sides of the back near the hind legs. The hemostatic forceps were blunt to separate the subcutaneous tissue and form pouches. A random sample was implanted in each pouch. The incisions were sutured, and the nude mice were put back into SPF environment for further feeding after they regained consciousness. After 4 weeks of experiment, all nude mice were killed by inhaling excessive carbon dioxide, the samples were taken out, dehydrated, paraffin-embedded, and sliced along the long axis of the tooth with a thickness of $5 \mu \mathrm{m}$. The spread pieces, patches and patches were baked at $58^{\circ} \mathrm{C}$ for 2 hours and then observed by $\mathrm{HE}$ staining (Baso Diagnostic, Inc., Zhuhai, Guangdong, China).

\subsection{Statistical analysis}

SPSS 24.0 was used for statistical analysis. Overall analysis of $\mathrm{pH}$ value, cell proliferation and gene expression in each group were analyzed by One-way ANOVA. LSD test was used for comparison between groups, and $P<0.05$ was considered statistically significant. 
190

191

192

193

194

196

197

198

199

200

201

202

203

204

205

206

207

208

209

210

211

212

Results

3.1 Morphology of MBG

MBG particles were observed by FE-SEM, as shown in Fig 1A. MBG was a spherical particle with regular morphology, rough surface and good dispersion. The particle size was sub-micron and the size was about 300-500nm. The mesoporous structure inside MBG particles was observed by TEM, as shown in Fig 1B. Regularly and uniformly distributed radial mesoporous structure can be seen inside MBG particles.

3.2 Physical and chemical properties of MBG

The $\mathrm{pH}$ change curve of the extracted solution with different concentrations of MBG and 45S5 for $24 \mathrm{~h}$ was detected by $\mathrm{pH}$ meter, and the results were shown in Fig $1 \mathrm{C}$. The $\mathrm{pH}$ of MBG extract increased with the increase of extraction time and reached the platform stage 6 hours later. When the concentration was $0.1 \mathrm{mg} / \mathrm{mL}$, the $\mathrm{pH}$ value of $\mathrm{MBG}$ extract increased from 7.40 to 7.70 in the first 1 hour, and then maintained at about 7.90 at the platform stage. The $\mathrm{pH}$ value of the 45S5 extract increased from 7.40 to 7.84 in the first 1 hour, then reached the platform stage and maintained at about 8.10 . The $\mathrm{pH}$ of $45 \mathrm{~S} 5$ group was significantly higher than that of $\mathrm{MBG}$ group at the same extraction time point $(P<0.05)$.

After $0.1 \mathrm{mg} / \mathrm{mL}$ MBG was extracted for 24 hours, the concentration of $\mathrm{Si}, \mathrm{Ca}$ and $\mathrm{P}$ ions in the solution was detected three times by ICP and the mean and standard deviation were provided (Table 2). As shown in Table 2, MBG could significantly improve the concentration of Si ions in the solution.

Table 2. Ion concentration of MBG extract 


\begin{tabular}{llll}
\hline & Si $($ ppm $)$ & $\mathrm{Ca}(\mathrm{ppm})$ & $\mathrm{P}(\mathrm{ppm})$ \\
\hline DMEM(BLK) & $0.076 \pm 0.002$ & $66.64 \pm 1.90$ & $27.61 \pm 0.77$ \\
$45 \mathrm{~S} 5$ & $12.14 \pm 1.29$ & $72.59 \pm 0.49$ & $27.48 \pm 0.23$ \\
MBG & $31.81 \pm 0.68$ & $51.54 \pm 2.45$ & $19.41 \pm 1.22$ \\
\hline
\end{tabular}

213

FE-SEM was used to observe the mineral deposition on the surface of MBG, as shown in Fig 1D. After soaking in SBF for 24 hours, a large number of short-stick mineral deposits could be seen on the surface of MBG particles. FTIR was used to detect and analyze the mineral components deposited on MBG surface, as shown in Fig 1E. The results showed that after MBG was immersed in SBF for 24 hours, a dispersion peak at $580 \mathrm{~cm}^{-1}$ on the FTIR was split into two peaks, which were located at 603 and $562 \mathrm{~cm}^{-1}$ respectively, representing a typical bimodal structure formed by the bending vibration of crystallized P-O bond. Hint: After MBG was soaked in SBF for 24 hours, the surface began to deposit minerals containing hydroxyapatite crystals. Fig 1F shows the XRD pattern of MBG soaked in SBF for 24 hours. After soaking for 1 day, the sample showed a diffraction peak at 2 at $26^{\circ}$ and $32^{\circ}$. According to the standard PDF card JCPDS-09-0432, the diffraction peak was hydroxyapatite (HA) diffraction peak. $26^{\circ}$ and $32^{\circ}$ correspond to (002) and (211) crystal surfaces respectively. This indicates that HA is formed on the surface of MBG.

\subsection{Effects of MBG on hDPCs proliferation and differentiation}

MTT assay was used to detect the number of living cells cultured with $0.1 \mathrm{mg} / \mathrm{mL} \mathrm{MBG}$ at different time points, and the results showed that there was no statistical difference between the cell proliferation curve after MBG treatment and the control group. It indicates that MBG $0.1 \mathrm{mg}$ $/ \mathrm{mL}$ does not affect hDPCs proliferation (Fig 2A). 
232

234

235

236

237

238

239

240

241

242

243

244

245

246

247

248

249

250

251

252

253

254

The expressions of ALP and DMP-1 of hDPCs dentin differentiation genes were detected by Real-Time PCR, and the results showed that the expressions of ALP and DMP-1 in MBG group were significantly higher than those in the control group at 7 days $(P<0.05)$. This indicated that MBG culture promoted the differentiation of hDPCs (Fig 2B).

3.4 MBG induced the formation of endodontic dentin complex in rats

4 weeks after in vivo transplantation, HE staining was shown in Fig 3. In the control group, there was mild inflammatory infiltration in pulp, and the number of pulp cells was small. The pulp section has a reddish fibre-like matrix with no visible hard tissue formation. In the pulp section of the MBG group in contact with the material, obvious hard tissue formation and regular structure can be seen, and a small amount of cavitation formed after MBG demineralization.

There was also a homogeneous layer of secondary dentine deposition near the pulp cavity. At high magnification, it can be seen that the mineralized matrix in the pulp section has dentin tubulelike structure. A few cells' cytoplasm protrude into the mineralized matrix to form the complex structure of pulp dentin.

\section{Discussion}

In this study, the surfactant $\mathrm{CTAB}$ was used as the template of mesoporous structure to prepare MBG with loose and divergent structure in the emulsion reaction system. It is characterized by uniform size, good dispersion and good in vitro mineralization induced activity. During the preparation of MBG, glassy sol particles were introduced into the CTAB induced system to polymerize inside their micelles. After volatilization of $\mathrm{CTAB}$, a pore structure is formed inside the glass particles[12]. Traditional silicate glass has a complete network structure formed by -Si- 
$255 \mathrm{O}-\mathrm{Si}$ - bridge oxygen bonds, which is relatively stable[13]. The incorporated $\mathrm{Ca}$ is introduced into 256 the system to interrupt the covalent network structure, changing the -Si-O-Si- bridge oxygen bond 257 into a non-bridging oxygen bond $-\mathrm{Si}-\mathrm{O}-\mathrm{M}^{+}\left(\mathrm{M}^{+}\right.$is the modified cation), forming a partially open 258 grid structure[14, 15]. The combination of the internal components becomes loose, the template

259

260

261

262

264

265

266

267

268

269

270

271

272

273

274

275

276

277

agent is easy to enter the interior, the connection between the small mesopores is opened, and the pore size is increased. In contact with liquid, there are more cations such as $\mathrm{Ca}^{2+}$ in contact with non-bridging oxygen and body fluids for rapid ion exchange, accelerating the formation of hydroxyapatite, thus showing better biocompatibility and bioactivity.

The average pore diameter of $\mathrm{MBG}$ is about $7.325 \mathrm{~nm}$. Kumar found that $\mathrm{MBG}$ with pore sizes of few nano meters exhibit favorable biocompatibility in vitro behavior and found to be promising candidate in the field of biomaterials including tissue regeneration and drug storage[16]. MBG has a relatively stable $\mathrm{pH}$. The cations released by $\mathrm{BG}$ can be replaced by $\mathrm{H}^{+} / \mathrm{H}_{3} \mathrm{O}^{+}$in aqueous solution through ion exchange to increase $\mathrm{pH}[17]$. The $\mathrm{Ca}^{2+}$ in $\mathrm{MBG}$ 24-hour extract is significantly lower than $45 \mathrm{~S} 5$, and the effect of increasing $\mathrm{pH}$ at a relatively high concentration $(1 \mathrm{mg} / \mathrm{mL})$ is also significantly lower than $45 \mathrm{~S} 5$. It was found that the $\mathrm{pH}$ value of 6.6 to 7.8 was more suitable for the growth of pulp cells. $\mathrm{pH}$ beyond this range can cause inflammatory responses in cells, leading to the death of pulp cells[18]. In this study, the effect of MBG on $\mathrm{pH}$ increase was significantly weaker than that of 45S5, suggesting that MBG has better biocompatibility with tissues and less influence on the growth of pulp cells in vivo.

MBG can release a higher level of Si, induce the expression of odontogenic genes in pulp cells and form a pulp dentin complex. A higher level of Si can promote the proliferation of dental pulp cells, promote the expression of cell surface integrin 2 and 1[19], promote the secretion of extracellular matrix of dental pulp, activate relevant downstream signaling pathways, and promote 
278 the differentiation of dental pulp cells into teeth[20]. The relatively stable weakly alkaline

279 environment provided by MBG also contributes to the odontogenic differentiation of pulp cells.

280 Similar results[21, 22] showed that MBG has good tissue induction, and has a faster degradation

281 rate compared with the traditional $45 \mathrm{~S} 5$ and $58 \mathrm{~S} \mathrm{BG}$, which is basically matched with the tissue 282 repair rate.

283 This study confirmed that MBG can promote the differentiation and mineralization of dental 284 pulp cells through in vitro and nude mouse subcutaneous experiments. At the same time, due to its 285 relatively large specific surface area, mesoporous materials may have a faster degradation rate 286 after contact with body fluids. Therefore, animal experiments may require a shorter observation 287 time to observe the early effects and degradation of the materials on dental pulp cells. On the other 288 hand, the large specific surface area of MBG demonstrates its excellent potential as a drug carrier 289 material. The MBG compound drug may be made into a bioactive pulp capping agent with antiinflammatory and antibacterial effects for more extensive application in vital pulp preservation.

Conclusions

MBG prepared by using $\mathrm{CTAB}$ as a template has relatively regular size and good dispersibility, large specific surface area, and relatively stable $\mathrm{pH}$ in solution. It can provide a suitable environment for the differentiation of human dental pulp cells into odontogenesis, and has a certain effect of inducing the formation of dental pulp-dentin complex, and has a good application prospect in the field of dental pulp damage repair and regeneration. 
300 We also thank Prof. Xiaofeng Chen and Dr. Yudong Wang from National Engineering

301 Research Center for Tissue Restoration and Reconstruction, Southwest University of Science and

302 Technology, Guangzhou 510006, China.

303 Funding

304 This work was supported by the National Natural Science Foundation of China (51372005). 
[1] Lalzawmliana V, Anand A, Roy M, et al. Mesoporous bioactive glasses for bone healing and biomolecules delivery. Materials science \& engineering C, Materials for biological applications. 2020;106:110180.

[2] Kargozar S, Singh R, Kim H, et al. "Hard" ceramics for "Soft" tissue engineering: Paradox or opportunity? Acta biomaterialia. 2020;115:1-28.

[3] Mi S, Dong Y, Gao X. [Effects of ionic-dissolution of sol-gel bioactive glasses on human dental pulp cells]. Beijing da xue xue bao Yi xue ban = Journal of Peking University Health sciences. 2012;44(1):39-42.

[4] Long Y, Liu S, Zhu L, et al. Evaluation of Pulp Response to Novel Bioactive Glass Pulp Capping Materials. Journal of endodontics. 2017;43(10):1647-50.

[5] Cui CY, Wang SN, Ren HH, et al. Regeneration of dental-pulp complex-like tissue using phytic acid derived bioactive glasses. Rsc Advances. 2017;7(36):22063-70.

[6] Hu Q, Li Y, Miao G, et al. Size control and biological properties of monodispersed mesoporous bioactive glass sub-micron spheres. Rsc Advances. 2014;4(43):22678-87.

[7] Fiume E, Barberi J, Verné E, et al. Bioactive Glasses: From Parent 45S5 Composition to Scaffold-Assisted Tissue-Healing Therapies. Journal of functional biomaterials. 2018;9(1).

[8] Yan X, Yu C, Zhou X, et al. Highly ordered mesoporous bioactive glasses with superior in vitro bone-forming bioactivities. Angewandte Chemie (International ed in English). 2004;43(44):5980-4.

[9] Hu Q, Jiang W, Li Y, et al. The effects of morphology on physicochemical properties, bioactivity and biocompatibility of micro-/nano-bioactive glasses. Advanced Powder Technology. 2018;29(8).

[10] Zhu L, Wang Y, Dong Y, et al. [Mesoporous nano-bioactive glass microspheres as a drug delivery system of minocycline]. Beijing da xue xue bao Yi xue ban = Journal of Peking University Health sciences. 2018;50(2):249-53. [11] Gronthos S, Mankani M, Brahim J, et al. Postnatal human dental pulp stem cells (DPSCs) in vitro and in vivo. Proceedings of the National Academy of Sciences of the United States of America. 2000;97(25):13625-30.

[12] Hu Q, Li Y, Zhao N, et al. Facile synthesis of hollow mesoporous bioactive glass sub-micron spheres with a tunable cavity size. Materials Letters. 2014;134(nov.1):130-3.

[13] Serra J, González P, Liste S, et al. Influence of the non-bridging oxygen groups on the bioactivity of silicate glasses. Journal of Materials Science Materials in Medicine. 2002;13(12):1221-5.

[14] Brauer, Delia S. Bioactive Glasses-Structure and Properties. Angewandte Chemie International Edition. 2015;54(14):4160-81.

[15] Tilocca A, Cormack A. Surface signatures of bioactivity: MD simulations of 45S and 65S silicate glasses. Langmuir : the ACS journal of surfaces and colloids. 2010;26(1):545-51.

[16] Kumar A, Aditya A, Murugavel S. Effect of surfactant concentration on textural characteristics and biomineralization behavior of mesoporous bioactive glasses. Materials science \& engineering $\mathrm{C}$, Materials for biological applications. 2019;96:20-9.

[17] Hench LL. The story of Bioglass. Journal of Materials Science Materials in Medicine. 2006;17(11):967.

[18] Hirose Y, Yamaguchi M, Kawabata S, et al. Effects of Extracellular pH on Dental Pulp Cells In Vitro. Journal of endodontics. 2016;42(5):735-41.

[19] Liu C, Hung C, Huang T, et al. Odontogenic differentiation of human dental pulp cells by calcium silicate materials stimulating via FGFR/ERK signaling pathway. Materials science \& engineering C, Materials for biological applications. 2014;43:359-66.

[20] Wu B, Kao C, Huang T, et al. Effect of verapamil, a calcium channel blocker, on the odontogenic activity of human dental pulp cells cultured with silicate-based materials. Journal of endodontics. 2014;40(8):1105-11.

[21] Wang S, Gao X, Gong W, et al. Odontogenic differentiation and dentin formation of dental pulp cells under nanobioactive glass induction. Acta biomaterialia. 2014;10(6):2792-803.

[22] Ravanbakhsh M, Labbaf S, Karimzadeh F, et al. Mesoporous bioactive glasses for the combined application of osteosarcoma treatment and bone regeneration. Materials science \& engineering $\mathrm{C}$, Materials for biological applications. 2019;104:109994. 


\section{Figure 1}

The morphology and physicochemical properties of 60S MBG

A. Particle scanning electron microscopy of MBG; B. Transmission electron microscopy of MBG; C. The extraction pH curve of MBG; D. Scanning electron microscopy after MBG immersion of simulated body fluids; E. The surface sediment infrared spectrum of MBG; F. The surface sediment X-ray diffraction pattern of MBG
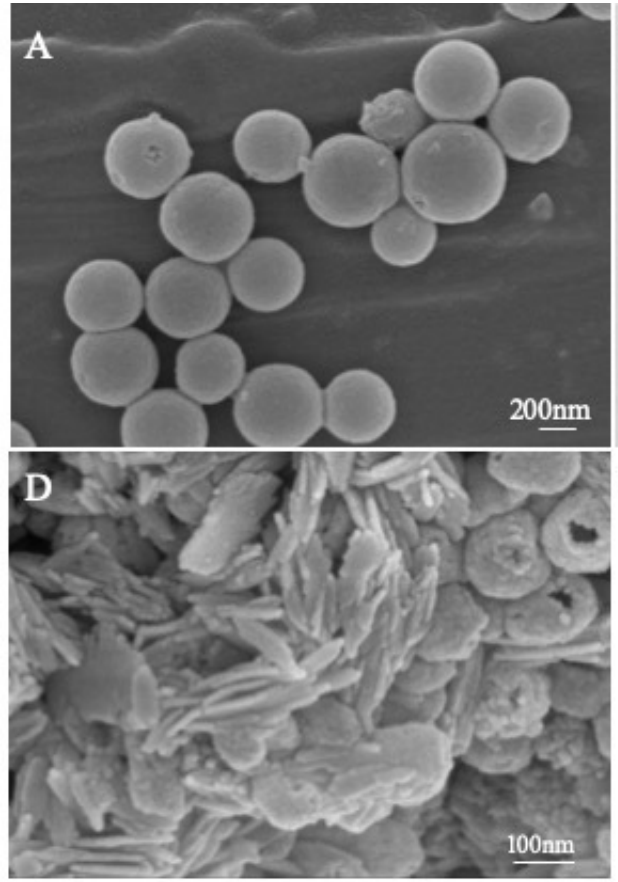

B

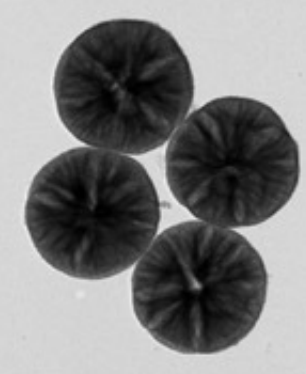

$\mathrm{E}$

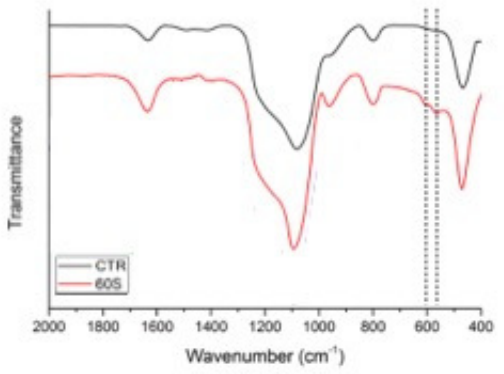

$\mathrm{C}$

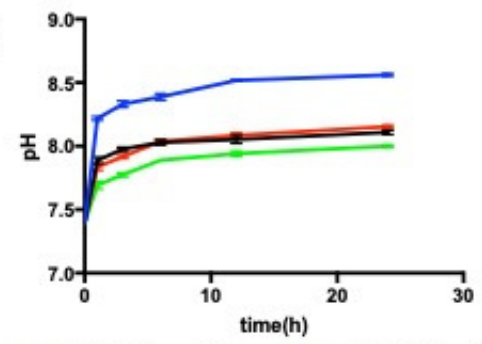

- $45 S 5(1 \mathrm{mg} / \mathrm{mL}) \quad-45 S 5(0.1 \mathrm{mg} / \mathrm{mL})$

$-60 S(1 \mathrm{mg} / \mathrm{mL}) \quad-605(0.1 \mathrm{mg} / \mathrm{mL})$

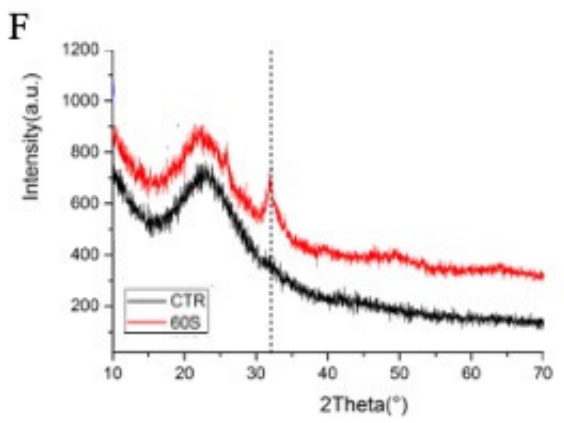


Figure 2

The effect of MBG on odontogenic differentiation of hDPCs

A. The effect of MBG on hDPCs proliferation detected by MTT; B. The effect of MBG on hDPCs odontogenic differentiation genes ALP and DMP-1 detected by RT-PCR. $* P<0.05$.

A

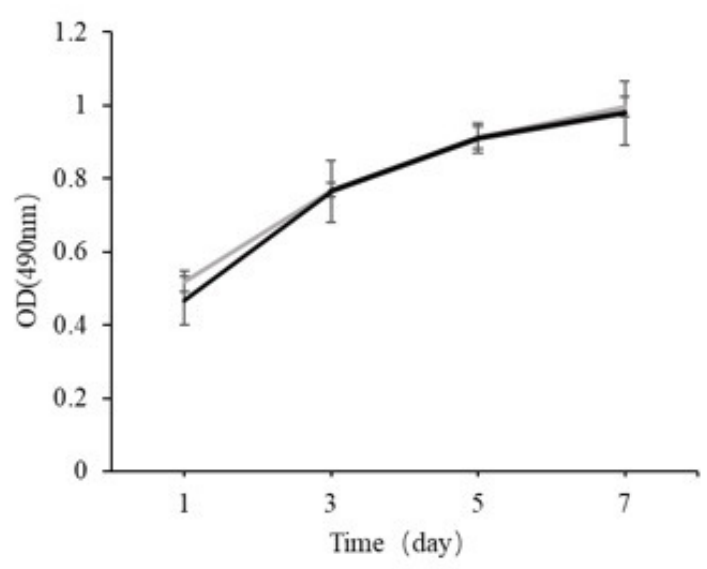

B

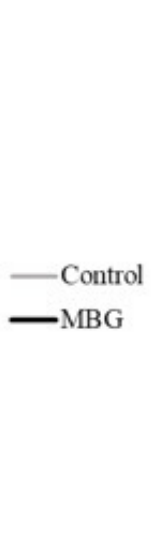

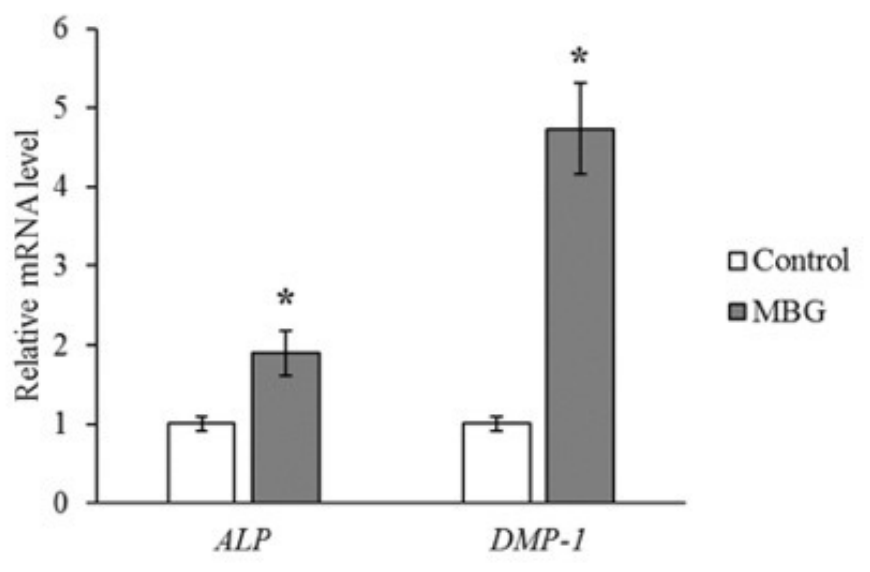




\section{Figure 3}

The formation of endodentine complex induced by MBG in rats

The formation of endodentine complex induced by MBG in rats (Note: the figure below is enlarged in the solid frame of the figure above. Abbreviation: D, dentin; P, pulp tissue; RD, reparative dentine, $\mathrm{DL}$, dentin tubule-like structure, $\mathrm{OL}$, osteoid dentin structure.)
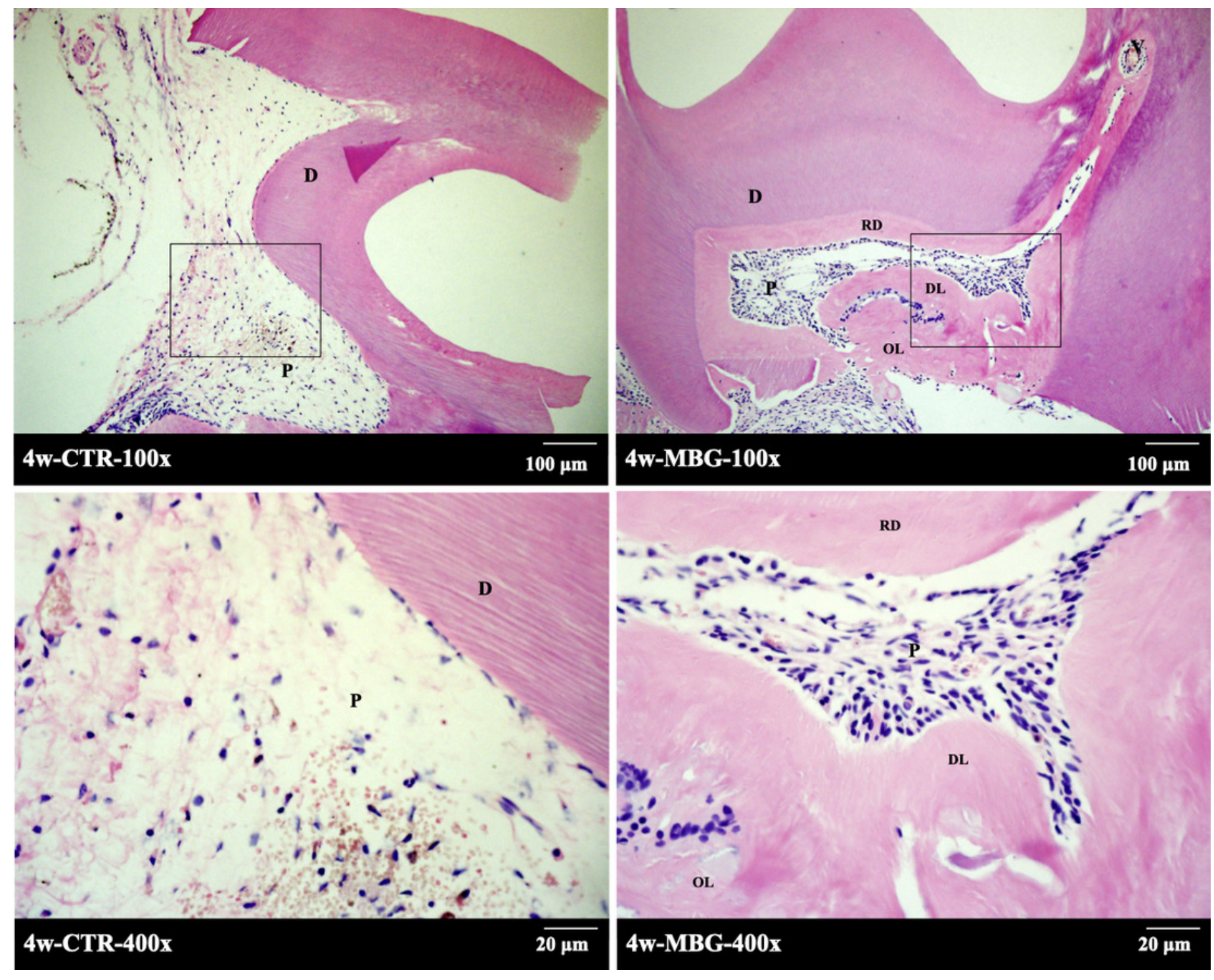